\title{
$\int$

Treatment of osteochondral lesions of the talus with autologous collagen-induced chondrogenesis: clinical and magnetic resonance evaluation at one-year follow-up

\author{
FEDERICO GIUSEPPE USUELLI ${ }^{1}$, MIRIAM GRASSI ${ }^{1}$, LUIGI MANZI ${ }^{1,2}$, VINCENZO GUARRELLA $^{1,3}$, \\ MICHELE BOGA ${ }^{1}$, LAURA DE GIROLAMO ${ }^{4}$ \\ 1 IRCCS Istituto Ortopedico Galeazzi, USPEC, Milano, Italy \\ 2 Seconda Università degli Studi di Napoli, Napoli, Italy \\ ${ }^{3}$ Università degli Studi di Milano, Milano, Italy \\ ${ }^{4}$ IRCCS Istituto Ortopedico Galeazzi, Laboratorio di Biotecnologie applicate all'Ortopedia, Milano, Italy
}

\begin{abstract}
Purpose: the aim of this study is to report the clinical and imaging results recorded by a series of patients in whom osteochondral lesions of the talus (OLTs) were repaired using the autologous collagen-induced chondrogenesis (ACIC) technique with a completely arthroscopic approach.

Methods: nine patients (mean age $37.4 \pm 10$ years) affected by OLTs (lesion size $2.1 \pm 0.9 \mathrm{~cm}^{2}$ ) were treated with the ACIC technique. The patients were evaluated clinically both preoperatively and at 12 months after surgery using the American Orthopaedic Foot and Ankle Society Ankle-Hindfoot Scale (AOFAS) and a visual analog scale (VAS). For morphological evaluation, the magnetic resonance observation of cartilage repair tissue (MOCART) score was used.

Results: the AOFAS score improved from $51.4 \pm 11.6$ preoperatively to $71.8 \pm 20.6$ postoperatively, while the VAS value decreased from $6.9 \pm 1.8$ to $3.2 \pm 1.9$. The mean MOCART score was $51.7 \pm 16.6$ at 12 months of follow-up; these scores did not directly correlate with the clinical results.

Conclusion: use of the ACIC technique for arthroscopic repair of OLTs allowed satisfactory clinical results to be obtained in most of the patients as soon
\end{abstract}

\footnotetext{
Corresponding Author:

Laura de Girolamo, MD

IRCCS Istituto Ortopedico Galeazzi, Laboratorio di Biotecnologie applicate all'Ortopedia

Via Galeazzi 4, 20161 Milano, Italy

E-mail: laura.degirolamo@grupposandonato.it
}

as one year after surgery, with no major complications or delayed revision surgery. ACIC is a valid and lowinvasive surgical technique for the treatment of chondral and osteochondral defects of the talus.

Level of evidence: therapeutic case series, level IV.

Keywords: ACIC, osteochondral lesion, arthroscopy, talus, MOCART.

\section{Introduction}

Osteochondral lesions of the talus (OLTs) are a frequent cause of ankle pain (1). The etiology of chondral and osteochondral defects is unclear $(2,3)$. The occurrence of traumatic injuries, such as ankle sprains and fractures, which can result in instability, has been proposed as the principal etiological factor, although trauma is not documented in all OLT cases (4-6).

Various systems, often based on imaging findings, have been developed for classifying osteochondral lesions. Berndt and Harty first classified OLTs according to findings on plain radiographs (7); much later (in the 1990s), Loomer et al. proposed a five-stage computed tomography classification system (8), while Hepple et al. (9) described a magnetic resonance imaging (MRI)-based staging system of talar dome injury and Ferkel et al. proposed an arthroscopic classification system (10).

Non-surgical management of OLTs is usually reserved for cases characterized by the presence of a nondisplaced osteochondral fragment; the most common treatments are nonsteroidal anti-inflammatory drugs, 
corticosteroids, and, more recently, hyaluronic acid (11) and autologous growth factor (platelet-rich plasma, PRP) injections $(11,12)$. Due to unsatisfactory results with non-operative management (13), surgical management is indicated for acute lesions, displaced fragments and osteochondral lesions refractory to non-operative treatments.

Although the optimal surgical technique for OLTs remains uncertain, biological surgical approaches based on bone marrow stimulation, such as abrasion, debridement, drilling and microfractures, have been extensively used in recent decades (14). These procedures are relatively inexpensive and low risk, and they allow satisfactory results in the short term (15-17). However, grafting techniques (e.g. the use of autografts, allografts and synthetic or natural grafts) may be required in cases with larger defects, and to allow more durable results $(18,19)$. In this context, matrixassisted bone marrow stimulation is one of the most innovative techniques for the treatment of osteochondral lesions, allowing a one-step surgical treatment. The matrices used in this kind of application are usually made from components of the cartilage extracellular matrix, in particular hyaluronic acid and collagen, in order to provide mesenchymal stem cells with a microenvironment conducive to the achievement of an effective cartilage regeneration process (20-22). These procedures are often performed with an open approach; they generally require malleolar osteotomies and extensive arthrotomy that may result in nonunion or malunion of the malleolus, ankle stiffness, and longer rehabilitation (23). The autologous collagen-induced chondrogenesis (ACIC) technique is used in association with injectable collagen filler and thus it allows the chondral and osteochondral defects to be treated completely arthroscopically $(22,24)$. One such filler, Cartifill $^{\mathrm{TM}}$ (Joint Biomaterials sas, Mestre, Italy) is an injectable scaffold made of atelocollagen isolated from porcine dermis, and nutrients essential for the maintenance of mesenchymal cells; it creates a stable matrix that, covering the cartilage defect, can be colonized by cells deriving from microfractures of the subchondral bone.

The aim of this study is to describe the clinical and the imaging outcomes at one-year follow-up of patients treated for OLTs with the ACIC technique.

\section{Methods}

From November 2012 to March 2013, 9 patients (6 males, 3 females) affected by OLT were consecutively treated with arthroscopic ACIC at our Institute.

All these patients presented the following indications for this kind of surgery: age between 18 and 55 years; presence of one isolated OLT (grade E or F according to Ferkel et al.) (10) with an area larger than $1.5 \mathrm{~cm}^{2}$ or at least one dimension $>15 \mathrm{~mm}$, and a depth $>1 \mathrm{~cm}$, as assessed by MRI; recurrent pain; swelling; occasional stiffness; a clicking sensation.

The study was approved by the institutional review board (IRB) and each patient gave his/her consent to participate to the study.

\section{Surgical cartilage repair technique}

The whole procedure was performed arthroscopically by the same senior surgeon. Once the presence and size of the defect had been confirmed, the Hintermann's spreader was placed percutaneously to distract the joint and allow exposure of the lesion. The damaged cartilage and scar tissue were removed, to create a regular-shaped site, building up shouldered borders when possible. Microfractures were performed using a chondral pick. At this point the intra-articular water was removed, the joint dried and the tourniquet deflated, in accordance with Volpi et al. (22), to promote the bleeding from the microfractures. Then, using a twoway syringe connected with a needle, Cartifill ${ }^{\mathrm{TM}}$ combined with fibrin glue (Tisseel ${ }^{\mathrm{TM}}$, Baxter, Deerfield, IL, USA), in a 1:1 ratio, was injected through the arthroscopic portal to cover the lesion. The Cartifill ${ }^{\mathrm{TM}}$ polymerized within 6-8 minutes.

Once the matrix appeared to be stable, the normal ankle range of motion (ROM) was assessed.

Postoperative management followed the general protocol for this kind of surgical treatment: no weight bearing was allowed for 6 weeks, and a very limited passive and active ROM for 2 weeks after surgery.

A strengthening and proprioceptive exercise program was prescribed $6 / 8$ weeks postoperatively, allowing the patients to participate in low-impact sports and activities such as swimming or cycling. Full activity was resumed, as suggested, over the 3-4 months after surgery. 


\section{Toints}

F. G. Usuelli et al.

$\sqrt{10}$

\section{Clinical and imaging evaluation}

The Articular Cartilage Imaging Group of the International Cartilage Repair Society (ICRS) defined MOCART, a set of pertinent variables for describing articular cartilage repair tissues after treatment using a biological repair technique (25); the MOCART assesses the degree of defect repair and filling, the border zone integration, the surface, structure and signal intensity of the repair tissue, the subchondral lamina and bone, adhesions and effusion, and it gives a score ranging from 0 to 100. The MOCART score was calculated on post-operative MRI 12 months after surgery by two independent orthopaedic surgeons blinded to the study. All MRI procedures were performed at 1.5T (MAGNETOM Avanto, Siemens Medical Solution, Forchein, Germany, gradient strength $45 \mathrm{mT} / \mathrm{m}$, slew rate 200 T/m/ms; MAGNETOM Espree, Siemens Medical Solution, Forchein, Germany, gradient strength 33 $\mathrm{mT} / \mathrm{m}$, slew rate $170 \mathrm{~T} / \mathrm{m} / \mathrm{ms}$ ), using standard imaging sequences and a dedicated extremity coil. Lesions were studied with the following imaging protocol: sagittal T1-weighted turbo spin-echo (TR $500 \mathrm{~ms}$, TE $9.2 \mathrm{~ms}$, thickness $3 \mathrm{~mm}$ ); axial T1-weighted turbo spin-echo (TR $500 \mathrm{~ms}$, TE $9.2 \mathrm{~ms}$, thickness $3 \mathrm{~mm}$ ); sagittal T2-weighted STIR short tau inversion recovery (TR $3990 \mathrm{~ms}$, TE $29 \mathrm{~ms}$, TI $160 \mathrm{~ms}$, thickness 3 $\mathrm{mm}$ ); axial proton density-weighted fat-saturated (TR $4120 \mathrm{~ms}$, TE $32 \mathrm{~ms}$, thickness $3 \mathrm{~mm}$ ); coronal proton density-weighted fat-saturated (TR $4120 \mathrm{~ms}$, TE 32 $\mathrm{ms}$, thickness $3 \mathrm{~mm}$ ); coronal T2-weighted turbo spinecho (TR 4500, TE 81, thickness $3 \mathrm{~mm}$ ).
The patients were evaluated clinically both preoperatively and 12 months after surgery; American Orthopaedic Foot and Ankle Society Ankle-Hindfoot Scale (AOFAS) and Visual Analog Scale (VAS) scores were recorded 12 months after surgery for each patient at the same time points. The AOFAS was used to evaluate function, pain and alignment, while the VAS appraised the severity of pain $(26,27)$. All the patients were clinically evaluated by the same clinician blinded to the study.

\section{Statistical analysis}

The statistical analysis was performed using the Matlab statistical toolbox version 2008 (MathWorks, Natick, MA, USA) for Windows (32-bit). All the results were expressed as mean values \pm standard deviation. Student's t test and the Pearson correlation coefficient $(\mathrm{R})$ were used for data analysis. A $\mathrm{p}$ value $<0.05$ was considered statistically significant.

\section{Results}

The 9 patients had a mean age of $37.4 \pm 10$ years (range 25-55) and a mean body mass index (BMI) of 25.5 \pm 3.3 (range 17.9-29.1). One had a central lesion of the talus, 6 a medial lesion and 2 a lateral lesion. Two lesions were contained and seven uncontained. The mean surface size of the OLT was $2.1 \pm 0.9 \mathrm{~cm}^{2}$ (range 1.15-3.87 $\mathrm{cm}^{2}$ ) (Tab. 1). There were no immediate major complications or any cases of delayed revision

Table 1. Clinical features of the patients.

\begin{tabular}{|c|c|c|c|c|c|c|c|}
\hline \multirow[t]{2}{*}{ PATIENT ID } & \multirow{2}{*}{$\begin{array}{c}\text { AGE } \\
\text { (years) }\end{array}$} & \multirow[t]{2}{*}{ BMI } & \multirow[t]{2}{*}{ LESION SIZE $\left(\mathrm{cm}^{2}\right)$} & \multicolumn{2}{|c|}{ AOFAS SCORE } & \multicolumn{2}{|c|}{ VAS SCORE } \\
\hline & & & & PRE-OP & POST-OP & PRE-OP & POST-OP \\
\hline 1 & 43 & 27.8 & 2.02 & 27 & 90 & 9 & 1 \\
\hline 2 & 35 & 28.3 & 1.54 & 49 & 55 & 8 & 3 \\
\hline 3 & 25 & 25.3 & 1.67 & 55 & 92 & 7 & 2 \\
\hline 4 & 29 & 17.9 & 1.72 & 44 & 80 & 8 & 4 \\
\hline 5 & 28 & 26.3 & 2.58 & 58 & 90 & 6 & 1 \\
\hline 6 & 48 & 29.1 & 1.53 & 53 & 63 & 5 & 6 \\
\hline 7 & 55 & 25.1 & 2.97 & 66 & 35 & 4 & 6 \\
\hline 8 & 33 & 23.8 & 3.86 & 48 & 87 & 6 & 2 \\
\hline 9 & 41 & 25.7 & 1.15 & 63 & 54 & 9 & 4 \\
\hline Mean & 37.4 & 25.5 & 2.1 & 51.4 & 71.8 & 6.9 & 3.2 \\
\hline SD & 10.0 & 3.3 & 0.9 & 11.6 & 20.6 & 1.8 & 1.9 \\
\hline
\end{tabular}

BMI, Body Mass Index; AOFAS Score, American Orthopaedic Foot and Ankle Society hindfoot scale; VAS, Visual Analogue Scale; pre-op, before surgery; post-op, after surgery. 
Table 2. MOCART evaluation of the patients' MRI scan.

\begin{tabular}{|c|c|c|c|c|c|c|c|c|c|c|}
\hline $\begin{array}{l}\text { PATIENT } \\
\text { ID }\end{array}$ & $\begin{array}{l}\text { Degree of } \\
\text { defect } \\
\text { repair } \\
\text { and filling of } \\
\text { the defect }\end{array}$ & $\begin{array}{l}\text { Integration } \\
\text { to border } \\
\text { zone }\end{array}$ & $\begin{array}{l}\text { Surface of } \\
\text { the repair } \\
\text { tissue }\end{array}$ & Adhesions & $\begin{array}{l}\text { Structure } \\
\text { of the } \\
\text { repair } \\
\text { tissue }\end{array}$ & $\begin{array}{l}\text { Signal } \\
\text { intensity } \\
\text { of the } \\
\text { repair } \\
\text { tissue }\end{array}$ & $\begin{array}{l}\text { Subchondral } \\
\text { lamina }\end{array}$ & $\begin{array}{l}\text { Subchondral } \\
\text { Subchondral }\end{array}$ & $\begin{array}{c}\text { Effusion } \\
\text { bone }\end{array}$ & $\begin{array}{c}\text { Total } \\
\text { MOCART } \\
\text { score }\end{array}$ \\
\hline 1 & 20 & 15 & 10 & 5 & 5 & 30 & 0 & 0 & 5 & 90 \\
\hline 2 & 15 & 10 & 5 & 0 & 0 & 10 & 0 & 0 & 5 & 45 \\
\hline 3 & 15 & 15 & 0 & 0 & 0 & 0 & 0 & 0 & 5 & 35 \\
\hline 4 & 10 & 10 & 5 & 5 & 5 & 10 & 0 & 0 & 5 & 50 \\
\hline 5 & 15 & 10 & 5 & 0 & 0 & 10 & 0 & 0 & 5 & 45 \\
\hline 6 & 15 & 15 & 5 & 5 & 0 & 10 & 0 & 0 & 5 & 55 \\
\hline 7 & 15 & 15 & 10 & 5 & 0 & 0 & 0 & 0 & 5 & 50 \\
\hline 8 & 20 & 10 & 10 & 5 & 0 & 10 & 0 & 0 & 5 & 60 \\
\hline 9 & 10 & 10 & 5 & 5 & 0 & 0 & 0 & 0 & 5 & 35 \\
\hline Mean & 15,0 & 12,2 & 6,1 & 3,3 & 1,1 & 8,9 & 0,0 & 0,0 & 5,0 & 51,7 \\
\hline SD & 3,5 & 2,6 & 3,3 & 2,5 & 2,2 & 9,3 & 0,0 & 0,0 & 0,0 & 16,6 \\
\hline
\end{tabular}

surgery. Although one patient showed clinical worsening (pain and functional scores), no indication for a second surgery was given. Pain relief was observed in 7 patients, whereas pain worsened in 1 and remained unchanged in the other; the VAS pain score ranged from a mean of $6.9 \pm 1.8$ preoperatively (range 4-9) to $3.2 \pm 1.9$ post-operatively (range 1-6) (n.s). The AOFAS score improved from a preoperative value of 51.4 \pm 11.6 (range 27-66) to a post-operative value of $71.8 \pm 20.6$ (range 35-92), although again the difference was not statistically significant. The patients had a mean MOCART score of 51.7 \pm 16.6 (range 35-90) at one-year follow-up (Tab. 2). The MRI studies showed that the regeneration process was still in progress, with satisfactory results in most of the patients (Fig. 1). A significant correlation was observed between AOFAS
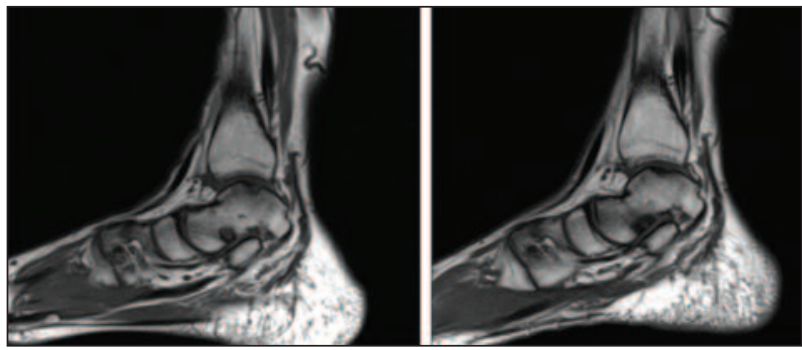

Fig. 1. Sagittal ankle MRI of a patient treated with the ACIC technique before (left) and after (right) surgery. and age $(\mathrm{R}=0.738, \mathrm{p}<0.05)$, whereas there were no significant correlations between VAS and age $(\mathrm{R}=0.671)$, BMI $(\mathrm{R}=0.40)$ or lesion size $\left(\mathrm{R}^{2}=0.04\right)$; similarly no correlation was found between AOFAS and BMI $(\mathrm{R}=0.019)$ or between AOFAS and lesion size $(\mathrm{R}=0.12)$. The MOCART score, too, was not significantly correlated with age $(\mathrm{R}=0.318)$, BMI $(\mathrm{R}=0.17)$, lesion size $(\mathrm{R}=0.329)$, VAS $(\mathrm{R}=0.268)$ or AOFAS (R=0.29) (Fig. 2).

\section{Discussion}

The current study reports the clinical and imaging outcomes of 9 patients treated with the ACIC technique for OLTs. Albeit referring to a small number of patient and a short follow up, our findings seem to demonstrate that ACIC is a safe technique that allows satisfactory clinical improvements to be obtained in most patients, particularly in terms of general satisfaction and quality of life. We are among the first to include a MOCART analysis in a clinical report on the application of ACIC in the talus; MOCART is an index of the quality of the regeneration process and is thus an important parameter for evaluating this kind of procedure (28).

A recent systematic review including 15 level I and II 

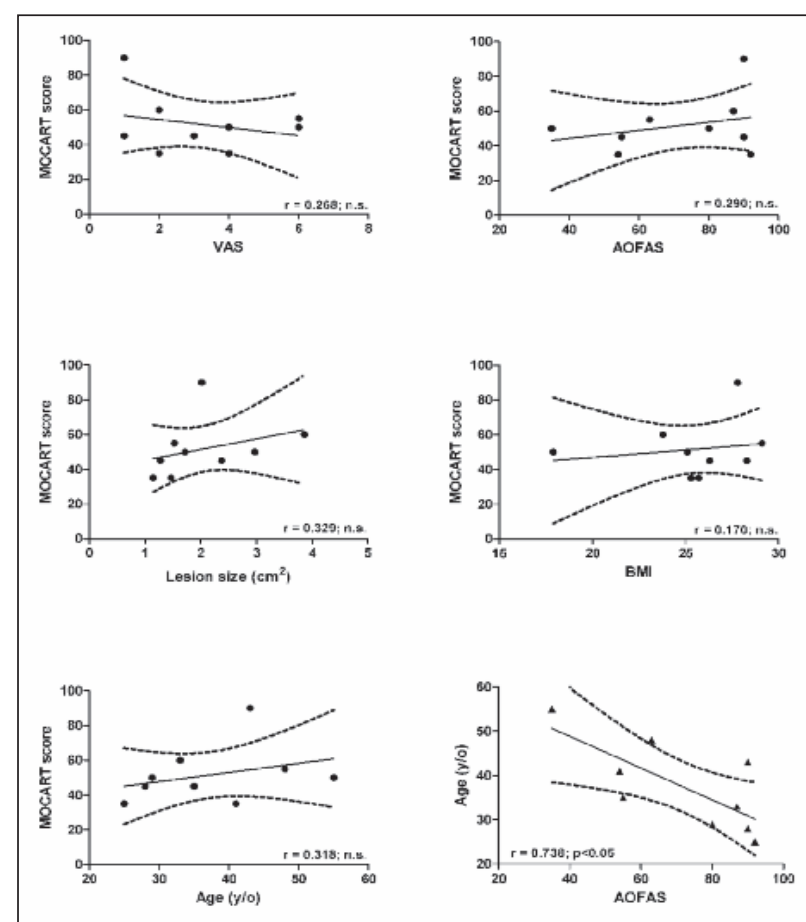

Fig. 2. Correlation of MOCART score with clinical outcomes and patients' characteristics. No significant correlation was found between MOCART and age, lesion size, VAS or AOFAS. A significant correlation between AOFAS score and age is shown.

studies reported that the use of bone marrow stimulation for chondral and osteochondral repair resulted in good clinical outcomes at short-term follow-up. However, beyond 5 years post-operatively, treatment failure could be expected (19), which suggests that the repair tissue obtained with the microfracture technique does not possess the same biological and biomechanical properties as the native tissue. For these reasons, new strategies for cartilage repair have been investigated in recent decades; among these, matrixassisted bone marrow stimulation techniques have been shown to be very promising: microfracture of the subchondral bone allows marrow chondroprogenitor cells to migrate into the lesion, and the matrix creates a regeneration "chamber" able to protect the blood clot and promote better tissue regeneration, mediated by these cells.

In the setting of ankle pathology, ACIC with Cartifill ${ }^{\mathrm{TM}}$ could be advantageous for the treatment of OLT due to its cytocompatibility (22) and low invasiveness; indeed, it is a fully arthroscopic technique, able to provide uniform cell distribution along the depth of the defect (22); furthermore, it is a readily available one-step surgical procedure, and thus limits costs. However, very few data are currently available on the clinical outcome and quality of the cartilage repair tissue obtained using this technique, in particular for the treatment of OLTs.

In our study, no patient undergoing the ACIC technique with Cartifill ${ }^{\mathrm{TM}}$ had major complications, including mobilization of the matrix, which confirms the existing literature data showing the safety of this approach and its lack of major complications $(22,29$, 30). As shown by the results of the assessment tools, the patients, on average, had noticeable improvements in terms of pain relief and functional outcomes at a mean one-year follow-up. One patient reported negative results after surgical treatment, probably due to his older age and larger lesion size, although his condition did not require any kind of revision surgery. Other two patients were not completely satisfied with the treatment, reporting a worsening of pain symptoms; in these cases, too, this was probably related to the initial large size of their lesions. Some Authors have indeed reported that lesion size and age are significant predictors of the outcome of arthroscopic treatment of OLTs $(19,31,32)$, with better results recorded in the presence of smaller defects and in younger patients. Our data are in line with those reported by Volpi et al., who treated 5 patients affected by osteochondral defects of the talus with Cartifill $^{\mathrm{TM}}$, and obtained a significant improvement in VAS pain scale (from 6.6 \pm 1.1 to $1.6 \pm 1.5$ ) and in AOFAS (from 53.8 \pm 15.1 to $86 \pm 9$ ) scores at 6 months after surgery (22). Wiewiorski et al. described the clinical and MOCART outcomes of 23 patients treated with the AMIC technique (with Chondro-Gide ${ }^{\circledR}$ ) for isolated OLT. At the time of follow-up (23 \pm 12 months), the AOFAS score had increased significantly from 60.3 to 90.9 , the mean VAS value had decreased significantly from 4.8 to 1.3 , and the mean MOCART score was 62.6 points (33). Valderrabano et al. treated 26 OLTs with autologous spongiosa graft harvested from iliac crest and a collagen I/III matrix (ChondroGide $^{\circledR}$ ). The AOFAS score improved from 60 to 89 and the VAS score from 5 to 1.6 at a mean follow-up of 31 months. The MOCART score after cartilage repair was 62 (21). The use of this kind of matrix can be enhanced by adding autologous biological stimuli, 
including bone marrow or platelet concentrate, able to participate in and improve the regeneration process. For example, Giannini et al. treated OLTs using hyaluronic acid membrane loaded with bone marrow concentrate. In their case series of 49 patients, the AOFAS score improved from 63.73 to 82.19 at 4 -year follow-up (20).

These one-step techniques make it possible to obtain clinical results similar to those obtained using twostage procedures such as matrix autologous chondrocyte implantation (MACI). In 18 patients with OLTs or osteochondral lesions of the tibial plafond undergoing MACI, the AOFAS score improved from 58.6 to 80.4 points at a mean follow-up of 24.5 months, and the MOCART score was 62.4 (34). Similarly, 30 patients affected by OLT were reported to have a mean AOFAS score that had improved from 36.9 to 83.9 at a mean follow-up of 45 months (21). In another study, Anders et al. applied the MACI technique in 22 consecutive patients: the AOFAS score improved from 70.1 to 95.3 points and pain intensity decreased from 5.7 to 0.9 at a mean 5-year follow-up, when the MOCART score was 83.8 (35). Lee et al. also showed that in patients treated with fibrin matrixmixed gel-type autologous chondrocyte implantation the average AOFAS score improved from 71 before surgery to 91 at 24 months, while the VAS score decreased from 5.8 before implantation to 2.1 at two years (36).

Although the functional outcomes after this kind of treatment are very important, so too is the quality of the repair tissue obtained, as the fibrocartilage produced by the repair process usually has lower biochemical and biological properties compared with the native cartilage. In this regard, the MOCART score, based on MRI findings, is considered a reliable index of repair tissue quality. The one-step techniques, including AMIC, have been shown to lead to variable MOCART results; our results showed an average score of around 50 points at one-year follow-up, and thus confirmed previous findings of matrix-assisted bone marrow stimulation techniques for the treatment of OLTs $(21,33)$.

Stelzeneder et al. described repair tissue obtained after application of the ACIC technique to treat knee chondral defects: in this study, the T2 values in the repair tissue had similar quantitative MRI properties to native hyaline cartilage, suggesting the formation of a hyaline-like repair tissue as early as one year after the treatment (30). These findings suggest that there is a need to assess longer-term results of ACIC used to treat OLTs, as this would provide more precise information about the efficacy of this approach in terms of the morphological tissue repair process.

Moreover, in our study no significant correlation was found between the MOCART score and the various clinical outcomes; this probably due, in part, to the small number of patients included. However, our findings are in line with other studies, which showed no correlation between MOCART and functional scores $(18,20,21,34,35)$. In any case, as the MOCART score was originally developed for chondral lesions of the knee joint $(28,37)$, it does not take into account certain peculiar features of the repair process occurring in the presence of OLTs. Delayed gadoliniumenhanced magnetic resonance imaging of cartilage (dGEMRIC), which is able to assess the presence of glycosaminoglycan in cartilage, would probably have been most suitable tool for assessing the quality of the repair tissue $(38,39)$. However, this technique requires the use of gadolinium as a contrast agent and for this reason our IRB refused to grant approval for its use in this study.

The main limitations of this study are the small number of patients and the lack of a control group. Also, a longer follow-up could have allowed us to observe more mature repair tissue, as it is known that the cartilage maturation process can last up to three years $(40,41)$. In conclusion, this study showed that the arthroscopic ACIC technique for the treatment of OLTs is a safe procedure allowing satisfactory results to be obtained with a minimally invasive surgical procedure. A larger number of patients and longer follow-up could further confirm these results.

\section{References}

1. Campbell CJ, Ranawat CS. Osteochondritis dissecans: the question of etiology. J Trauma. 1966;6:201-221.

2. Uozumi H, Sugita T, Aizawa T, et al. Histologic findings and possible causes of osteochondritis dissecans of the knee. Am J Sports Med. 2009;37:2003-2008.

3. Yonetani $\mathrm{Y}$, Matsuo $\mathrm{T}$, Nakamura $\mathrm{N}$, et al. Fixation of detached osteochondritis dissecans lesions with bioabsorbable pins: clinical and histologic evaluation. Arthroscopy. 2010;26:782-789.

4. Hermanson E, Ferkel RD. Bilateral osteochondral lesions of the talus. Foot Ankle Int. 2009;30:723-727. 
5. Woods K, Harris I. Osteochondritis dissecans of the talus in identical twins. J Bone Joint Surg Br. 1995;77:331.

6. Usuelli FG, Mason L, Grassi M, et al. Lateral ankle and hindfoot instability: a new clinical based classification. Foot Ankle Surg. 2014;20:231-236.

7. Berndt AL, Harty M. Transchondral fractures (osteochondritis dissecans) of the talus. J Bone Joint Surg Am. 1959;41A:988-1020.

8. Loomer R, Fisher C, Lloyd-Smith R, et al. Osteochondral lesions of the talus. Am J Sports Med. 1993;21:13-19.

9. Hepple S, Winson IG, Glew D. Osteochondral lesions of the talus: a revised classification. Foot Ankle Int. 1999;20:789-793.

10. Ferkel RD, Cheng MS, Applegate GR. Osteochondral lesions of the talus: a radiologic and surgical comparison. Presented at the Annual Meeting of the American Academy of Orthopaedic Surgeons, Orlando, FL. 1995.

11. Mei-Dan O, Carmont MR, Laver L, et al. Platelet-rich plasma or hyaluronate in the management of osteochondral lesions of the talus. Am I Sports Med. 2012;40:534-541.

12. Engebretsen L, Steffen K, Alsousou J, et al. IOC consensus paper on the use of platelet-rich plasma in sports medicine. $\mathrm{Br}$ J Sports Med. 2010;44:1072-1081.

13. Zengerink M, Struijs PA, Tol JL, et al. Treatment of osteochondral lesions of the talus: a systematic review. Knee Surg Sports Traumatol Arthrosc. 2010;18:238-246.

14. Takao M, Uchio Y, Kakimaru H, et al. Arthroscopic drilling with debridement of remaining cartilage for osteochondral lesions of the talar dome in unstable ankles. Am J Sports Med. 2004;32:332-336.

15. Becher $\mathrm{C}$, Thermann $\mathrm{H}$. Results of microfracture in the treatment of articular cartilage defects of the talus. Foot Ankle Int. 2005;26:583-589.

16. Clanton TO, Johnson NS, Matheny LM. Outcomes following microfracture in grade 3 and 4 articular cartilage lesions of the ankle. Foot Ankle Int. 2014;35:764-770.

17. Kon E, Filardo G, Berruto M, et al. Articular cartilage treatment in high-level male soccer players: a prospective comparative study of arthroscopic second-generation autologous chondrocyte implantation versus microfracture. Am J Sports Med. 2011;39:2549-2557.

18. Giannini S, Battaglia M, Buda R, et al. Surgical treatment of osteochondral lesions of the talus by open-field autologous chondrocyte implantation: a 10-year follow-up clinical and magnetic resonance imaging T2-mapping evaluation. Am J Sports Med. 2009;37 Suppl 1:112S-118S.

19. Goyal D, Keyhani S, Lee EH, et al. Evidence-based status of microfracture technique: a systematic review of level I and II studies. Arthroscopy. 2013;29:1579-1588.

20. Giannini S, Buda R, Battaglia M, et al. One-step repair in talar osteochondral lesions: 4-year clinical results and t2-mapping capability in outcome prediction. Am J Sports Med. 2013;41: 511-518.

21. Valderrabano V, Miska M, Leumann A, et al. Reconstruction of osteochondral lesions of the talus with autologous spongiosa grafts and autologous matrix-induced chondrogenesis. Am J Sports Med. 2013;41:519-527.

22. Volpi P, Bait C, Quaglia A, et al. Autologous collagen-induced chondrogenesis technique (ACIC) for the treatment of chondral lesions of the talus. Knee Surg Sports Traumatol Arthrosc. 2014;22:1320-1326.

23. Verghese N, Morgan A, Perera A. Osteochondral lesions of the talus: defining the surgical approach. Foot Ankle Clin. 2013;18:49-65

24. Abelow SP. New advances in the treatment of cartilage injuries in the ankle. Presented at the Annual Fall Meeting of the
Arthroscopy Association of North America, Palm Desert, Calif. 2004;2-5.

25. Goebel L, Zurakowski D, Müller A, et al. 2D and 3D MOCART scoring systems assessed by $9.4 \mathrm{~T}$ high-field MRI correlate with elementary and complex histological scoring systems in a translational model of osteochondral repair. Osteoarthritis Cartilage. 2014;22:1386-1395.

26. Madeley NJ, Wing KJ, Topliss C, et al. Responsiveness and validity of the SF-36, Ankle Osteoarthritis Scale, AOFAS Ankle Hindfoot Score, and Foot Function Index in end stage ankle arthritis. Foot Ankle Int. 2012;33:57-63.

27. Carlsson AM. Assessment of chronic pain. I. Aspects of the reliability and validity of the visual analogue scale. Pain. 1983; 16:87-101

28. Marlovits S, Singer P, Zeller P, et al. Magnetic resonance observation of cartilage repair tissue (MOCART) for the evaluation of autologous chondrocyte transplantation: determination of interobserver variability and correlation to clinical outcome after 2 years. Eur J Radiol. 2006;57:16-23.

29. Shetty AA, Kim SJ, Bilagi P, et al. Autologous collagen-induced chondrogenesis: single-stage arthroscopic cartilage repair technique. Orthopedics. 2013;36:e648-652.

30. Stelzeneder D, Shetty AA, Kim SJ, et al. Repair tissue quality after arthroscopic autologous collagen-induced chondrogenesis (ACIC) assessed via T2* mapping. Skeletal Radiol. 2013; 42:1657-1664.

31. Becher C, Driessen A, Hess T, et al. Microfracture for chondral defects of the talus: maintenance of early results at midterm follow-up. Knee Surg Sports Traumatol Arthrosc. 2010; 18: 656-663.

32. Choi WJ, Kim BS, Lee JW. Osteochondral lesion of the talus: could age be an indication for arthroscopic treatment? Am J Sports Med. 2012;40:419-424.

33. Wiewiorski M, Miska M, Kretzschmar M, et al. Delayed gadolinium-enhanced MRI of cartilage of the ankle joint: results after autologous matrix-induced chondrogenesis (AMIC)-aided reconstruction of osteochondral lesions of the talus. Clin Radiol. 2013;68:1031-1038.

34. Aurich M, Bedi HS, Smith PJ, et al. Arthroscopic treatment of osteochondral lesions of the ankle with matrix-associated chondrocyte implantation: early clinical and magnetic resonance imaging results. Am J Sports Med. 2011;39:311-319.

35. Anders S, Goetz J, Schubert T, et al. Treatment of deep articular talus lesions by matrix associated autologous chondrocyte implantation--results at five years. Int Orthop. 2012;36:22792285.

36. Lee KT, Kim JS, Young KW, et al. The use of fibrin matrixmixed gel-type autologous chondrocyte implantation in the treatment for osteochondral lesions of the talus. Knee Surg Sports Traumatol Arthrosc. 2013;21:1251-1260.

37. Domayer SE, Welsch GH, Dorotka R, et al. MRI monitoring of cartilage repair in the knee: a review. Semin Musculoskelet Radiol. 2008;12:302-317.

38. Burstein D, Velyvis J, Scott KT, et al. Protocol issues for delayed Gd(DTPA)(2-)-enhanced MRI (dGEMRIC) for clinical evaluation of articular cartilage. Magn Reson Med. 2001; 45:36-41.

39. Samosky JT, Burstein D, Eric Grimson W, et al. Spatially-localized correlation of dGEMRIC-measured GAG distribution and mechanical stiffness in the human tibial plateau. J Orthop Res. 2005;23:93-101.

40. Recht M, White LM, Winalski CS, et al. MR imaging of cartilage repair procedures. Skeletal Radiol. 2003;32:185-200.

41. Verstraete KL, Almqvist F, Verdonk P, et al. Magnetic resonance imaging of cartilage and cartilage repair. Clin Radiol. 2004;59:674-689. 